\title{
Towards a smart city: an integrative spatial perspective on energy transition
}

\author{
Filip Gulan, Maros Finka \\ SPECTRA Centre of Excellence EU, Slovak University of Technology in Bratislava, \\ Vazovova 5, 81243 Bratislava, Slovakia \\ \{filip.gulan, maros.finka\} estuba.sk
}

\begin{abstract}
The shift towards sustainable use of renewable energy accompanied by noteworthy improvements in energy efficiency are considered as fundamental elements of energy transition, essential to the "smart city" concept as well. However, framing the role of the sustainable energy development in a mono-functional way does not allow for to use efficiently its multifunctional potential linked to sustainable development. As a result, the disconnection between energy and broader spatial and urban development agenda underplays the importance of potential synergy-effects between renewable energy production and- use and its localized context. The guiding narrative of this paper is that single although innovative solutions are insufficient and poor integration of sustainable energy-related initiatives creates barriers to achieve sustainability and limits potential synergies with their spatial context. Therefore, new demands on integrative and spatially sensitive approaches to the renewable energy development appear often catalysed by the implementation of the smart city concept and subsequent evolution of energy systems towards smart grids. This paper attempts to examine possible approaches to better understand how renewable energy systems and initiatives emerge, how to maintain and enhance them, and under which conditions they co-evolve with their unique context in a more structured and productive way towards the vision of smart city.
\end{abstract}

Keywords: energy transition, niche management, renewable energy, spatial planning, smart city

\section{Introduction}

The development and implementation of the smart city concept seems to one of the dominant issues in current urban agenda across Europe undergoing substantial shift from the high-tech based approach to the comprehensive concept of smart management of the resources (natural and human resources, technologies, knowledge...) of knowledge-based urban community. Energy, inherent part of every smart city concept is one of the most critical issues facing the EU today, being a headline issue of the climate change policy discussions as only few activities affect environment and economy as much and as continually as the production and use of energy. The more and more acute need of shifting towards more sustainable energy (SE) systems manifested by climate change and ever growing energy demand is 
considered as one of the major societal goals at global level. Innovations in the energy field together with broader and sustainable use of "clean energy" such as renewable energy sources (RES) accompanied by noteworthy improvements in energy efficiency are considered as one of the essential pillars of the climate change adaptation and mitigation efforts, also driving developments towards more sustainable cities - smart cities. While frequently used, the notion of "energy transition" towards more energy systems lacks a widely accepted definition as well as common grounds in terms of its implementation are absent (Loorbach et al. 2008; Araújo 2014). It seems evident that energy transition requires a fundamental, structural change in the way how we use and think about energy and it will take long time to unfold. This will most likely require re-organizing spatial structures, responsible institutions, governance structures and even much more, what in turn creates also new demands on novel, smart energyconscious approaches offered by spatial planning.

Small-scale, decentralized and community-driven SE initiatives are widely acknowledged to be a desirable feature of low carbon future, however, they face a range of challenges in the context of their integration into the physical and socioeconomic landscape which goes far beyond the municipal or regional competences. Framing the role of the SE concepts in a mono-functional way does not allow efficient use the multifunctional potential of SE (social, economic, environmental...) at multiple scale levels (local, regional, national etc.). This results in the disconnection between energy and broader spatial development agenda, what also underplays the role of potential synergy-effects linked to implementation of SE concepts. Only the development and implementation of SE territorial concepts with emphasis on renewable energy deployment can react more properly on many profound questions about its benefits, limitations and negative impacts, which are necessarily associated with such "energy transition" in spatial contexts. Moreover, a transition towards sustainable energy can be envisioned as a long-term process that involves reconfiguring of dynamic spatial patterns of socio-economic and ecological landscape, what has often been neglected in relation to spatial development.

If we consider energy systems as complex socio-technical systems, the emergence and development of more sustainable energy systems will inevitably imply a careful consideration of their complex relationships with the territory along with their dynamics. Given this context, the notion of supra-local (regional) development is often under-appreciated when it comes to the development of SE and vice versa, so the integrative solutions and potential synergy between both domains are frequently omitted. However, cultural and political-economic factors co-evolve with changes to the quality, location, and environmental impact of energy resources and extensive changes in the energy mix have often underpinned social and geographical change (Jiusto 2009). Therefore, one of the assumptions guiding reported research is that "weak" spatial-structural and socio-economic integration of energy-related initiatives creates barriers to their potential synergy with sustainable development of the territory. On this basis, the capacity of local actors, communities, municipalities and regions to create, maintain or adopt innovative SE practices and translate them into the sustainable development is perceived as once of the major spatially embedded "qualities" to be followed by energy-conscious spatial planning that serves as a "toolkit" for making our settlements smarter. 


\section{Make energy smart, but in time and space}

"Smart city" has gained its relevancy as a concept or a vision for a more sustainable development of cities and urban areas, yet has no universally accepted definition. Smart city is envisioned as having a high quality of life by excelling in multiple key areas - such as economy, mobility, environment, governance and, of course, energy - with improved efficiency of services to meet peoples' current and future needs. Considering that this concept inherently implies a transition (a widespreading and systemic innovation reshaping our cities), this paper suggests that it might be useful to learn from the transition management (TM) theory. In this context, TM provides a systemic view on change at all levels, its dynamics and complexity and thus exceeds "usual" smart city frameworks. TM provides a useful framework for considering the temporal aspects of (energy) transitions largely, but renders to be rather fuzzy on how it relates to other dimensions of space such as territorial or administrative spaces and their typologies (Smith et al. 2010). Therefore, we attempt to take the perspective of spatial planning on the smart city-energy transition continuum in order to explore new insights on renewable energy development at the interface between local and regional level.

Spatial planning can be described as largely public-sector-led repertoire of activities to influence the future spatial distribution of activities, to enhance the integration between different sectors, to create a more rational territorial organization of land uses including the linkages between them, to balance demands for development with the need to protect the environment, and to achieve social and economic objectives. Spatial planning is regarded a key instrument for establishing long-term sustainable frameworks for social, environmental and economic development (European Commission 1999; United Nations 2008) and thus relates also to the concept of smart city. Arguably, spatial planning has for some time been ignorant of the complexity and traditionally has focused on well-defined and tame problems, operating within an atemporal framework (De Roo et al. 2012). Often, solutions for the past problems has been sought rather than looking for flexibility and adaptability enhancing solutions. Therefore, making the connection between spatial planning and TM appears to be logical in order to foster their synergy as well as to address and at least minimize their shortcomings in relation to smart energy transition.

This can be the ground to provoke and challenge our understanding of the role of spatial planning in the context of smart city concepts and "smart energy transition", considered as a concept relying heavily on automation, information and communications technology (ICT), driven by both social and technological innovations. We need to place stronger emphasis on the relationship between spatial development and renewable energy development at the local and supra-local level (e.g. urban area - a city, its neighbourhoods and hinterland). In an attempt to bring complexity-based "transitions" science on boards, the idea of socio-technical energy systems (e.g. including smart grids) considered as complex adaptive systems is adopted. Under such circumstances, "hard infrastructure" for energy should be equally important in planning as dealing with non-technical aspects, which in turn involves building of soft infrastructure capacities. Crucial questions in this context are linked to the possibilities to rethink the nature and character of sustainable and low- 
carbon (urban) development processes (e.g. smart city initiative) in a more productive way forward in the context of spatial planning practice.

\section{Theoretical contexts}

Among other things, changing nature and framing of spatial planning across all levels can be attributed to the recognition of the complexity, uncertainty and irreversibility, for example, demonstrated by the climate change awareness and its relationship to energy use and sustainable development (Davoudi et al. 2009).

The place-based approach towards the sustainable energy systems based on the broader use of renewable energy can be built upon their territoriality and ability to build on specialized supra-local (regional) assets - efficient use of territorial capital implying careful utilization of available RES while considering their ubiquitous spatiality. The various forms of spatiality, territoriality, and decentralised character of RES constitute the premise of (relational) proximity between people, settlements and renewable energy technologies. This consideration becomes increasingly relevant in order to inform the spatial planning interventions more comprehensively and efficiently (Basta et al. 2012; Wolsink 2013).

Indeed, the fact that RES have substantially different characteristics compared to fossil or nuclear fuels needs to be taken into account. While RES as one of the most common alternative energy source are projected to increase in the long run ${ }^{1}$, electricity supply based on these spatially dispersed and (in many cases) variable source of energy requires substantial land resources and their development involves complex reorganisation of the territory. The consideration of the fundamental differences in the socio-spatial distribution and socio-economic properties related to RES in contrast to high-carbon energy resources (fossil fuels) seems crucial.

Along with the concept of smart grids, RES are deemed as a viable alternative to the conventional, rather centralised energy systems based on the fossil-fuels. However, incumbent high-carbon energy systems with their underlying infrastructure (understood in a broad sense) have evolved gradually over decades and thus are strongly established and can prove resilient to changes. And in order to meet the basic goals of a smart city, it is evident that traditional power systems will need to undergo a complex, gradual transition towards a more sustainable, more decentralized sociotechnical systems that will be indeed more "smart".

One of the practical responds to current challenges within the sustainable energy research and practice that has been largely adopted also in the concept of smart city is the ongoing evolution towards smart grids ${ }^{2}$. Basically, smart grids are envisioned as

\footnotetext{
${ }^{1}$ Also in the short term, referring to the 2020 Climate and Energy Package which introduced three key objectives: a $20 \%$ reduction in greenhouse gas emissions, a $20 \%$ share of renewables in total energy consumption and a $20 \%$ improvement in the EU's energy efficiency by 2020 (Commission \& the Commission 2010), Renewable energy Directive, Energy Efficiency Directive.

${ }^{2}$ For instance see also Giordano et al. 2011; European Innovation Partnership 2013; Kempener et al. 2013;
} 
socio-technical energy networks, where consumers become more autonomous - they become prosumers (e.g. they produce energy from RES, supply the grid and consume electricity while possessing improved information, control and choice) and interact with the grid (e.g. via smart meters) - enabling a two-way flow of energy and information. The main idea behind smart grid solutions is that they are designed to counteract the natural disadvantages of RES through improved grid integration and interaction between actors (market, generation, distribution and transmission utilities, end-users, consumers etc. are integrated particularly through ICT). The potential benefits associated with smart grids are in line with the idea of smart city: they are designed to bring improved grid stability, security, efficiency and reliable integration of RES that are largely variable in time and space at both large and smaller scales (Giordano et al. 2011; Kempener et al. 2013). In this regard, the need for careful consideration of the relationship between renewable energy, its potential integration within the (smart) power grid and their localised context needs to be taken into account and investigated in more detail (Solomon et al. 2003; Bagliani et al. 2010).

A "technical" perspective on development, which often dominates in our planning approaches, proves to be seriously limited and rather ossified when it comes to dealing with the complex web of relationships between actors and networks in physical, socio-economic, institutional environment, for example in the case of dealing with energy systems. In fact, if we look at energy systems as being an integral part of our society (socio-technical system), we also might assume that it will be more feasible to frame the phenomena of renewable energy development in a way that its social and spatial embeddedness are considered in planning (Pasqualetti 2011; Stremke \& van den Dobbelsteen 2012; Bridge et al. 2013). On this basis, we suggest to approach energy systems from the complex adaptive system's perspective. In this regard, complex systems theory proves to be highly relevant for understanding of how complex, socio-technical energy systems evolve and continuously adapt to changing internal and external conditions as we are witnessing in everyday reality. Arguably, this perspective can promote interesting ways to frame the development of sustainable energy systems (i.e. renewable energy) and their (spatial) integration. Focus on the build-up processes of indigenous energy innovation capabilities for low-carbon development as part of "energy transition" where renewable energy and technology are perceived as active elements in the territory, offers potential for interventions that go far beyond business-as-usual planning methodologies.

Management of different activities to "set-up" processes related to the energy transition towards more sustainable energy can learn and draw inspiration from TM approach. Interestingly, TM is built upon the key notions of complex systems theory and governance based on complexity and uncertainty in order to guide and influence the course and pace of complex systems change. TM literature generally assumes that dealing with persistent, wicked problems such as climate change and energy transition is long-term and requires transitions. Moreover, it points out rather consistently that directing of such complex changes can be supported substantially by insights into general patterns of complex systems dynamics. In other words, TM attempts to overcome the conflict between long-term imperatives and short-term concerns" (Kemp \& Loorbach 2006). That being said, "transitions" can be understood as fundamental changes in the structure, culture, and practices of societal systems that cannot be controlled but might be influenced and guided - also with the assistance of 
TM. Consideration that energy transition consists of different phases is in place so the fact that different transition phases will probably require different strategies needs to be taken into account (Loorbach 2010; Rotmans et al. 2001a; Rotmans \& Loorbach 2008). At this point, we argue that bringing complexity-based, temporal perspective of TM together with "spatiality" of spatial planning can enrich spatial development practice and improve our understanding of how we can put our cities on a "smarter and sustainable" trajectory.

Hence, the theoretical premise is that bridging the gap between current planning and governance of energy transition (TM) in relation to the broader spatial-physical and socio-economic integration of RES into the territory can allow for more synergy and thus offer potential answers to these questions. This paper intends to argue that synergy stimulated by co-evolution between sustainable energy systems and their localized and supra-local context embodies the very idea of the smart city concept- a synergy between technology, environment and society.

\section{Adding a spatial dimension to managing energy transition}

The multi-level perspective has been used to describe and unfold structural innovations in socio-technical systems, or in other words, transitions to sustainability in socio-technical systems ${ }^{3}$. From the socio-technical perspective, technology is seen as "being formed by, and embedded within, particular economic, social, cultural and institutional structures and systems of beliefs" (Berkhout et al. 2004, p.5). In fact, the energy system can be described as "all actors and artefacts that together produce the societal function of energy" (Verbong \& Loorbach 2012, p.9). In practice, the socialtechnical perspective can be illustrated at the example of social barriers related to the development of renewable energy, which can take many forms. The impacts of RES deployment on sustainability, individuals, communities and landscapes have often been underestimated yet the main focus has been on "hardware" solutions and their "economy". Such approach has often proved insufficient in terms of the integration of energy initiatives at multiple scales. Hence, a more inclusive approach which is prone to acceptance by the local society and less vulnerable for failure has been advocated elsewhere ${ }^{4}$. The emerging field of energy transitions presents us with interesting frameworks for guided change of socio-technical systems. These insights are particularly relevant in terms of our focus on the development and integration of sustainable energy systems. In this context, the multi-level perspective on transitions takes into account that nonlinear processes of change result from the interplay of developments at three qualitatively different scale levels and attends to the dynamic relationship between them:

\footnotetext{
${ }^{3}$ As in Rip \& Kemp 1998; Geels \& Kemp 2000; Geels 2002b; Geels 2011; Berkhout et al. 2004; Byrne et al. 2011; Smith et al. 2010

${ }^{4}$ For example in Pasqualetti 2011; Stremke \& van den Dobbelsteen 2012; Wolsink 2013
} 
- Micro level of niches, where innovations, norms, practices, alternatives and novelties emerge and eventually can form the seed for systemic change ${ }^{5}$

- Meso level, occupied by a (socio-technical) regime. Regime can be described as the space of established practices and associated rules that form the stability of the socio-technical system ${ }^{6}$

- Macro level, or (socio-technical) landscape. The exogenous level of landscape refers to overall societal setting (worldviews, paradigms) in which processes of change occur. Socio-technical landscape represents a wider context in which a regime and niches are embedded ${ }^{7}$

The multi-level perspective as a leading theoretical framework on transitions maps the entire transition process, which is seen as a result of alignments between multiple developments at different levels, and brings innovative agency into play. The intermediate level of a regime is central in a way that the transition (systemic change) is viewed as a shift from one socio-technical regime to another, whereas both niche and landscape levels are characterized by their interactions with the regime level.

Hypothetically, a transition from centralized energy systems (e.g. based on fossil fuels and nuclear power) to a more differentiated and decentralized energy systems (e.g. relying largely on RES) can be envisioned as one of the alternative visions within the TM framework ${ }^{8}$. In this context, the multi-level perspective can help to map, analyse and explain how the variety of sustainable energy initiatives may emerge. That being said, TM framework holds the potential to provide spatial planning with deeper understanding on how can the build-up momentum connected to the niche level be facilitated, steered and influenced, which is in line with the focus of this research on the local-regional interface. The idea that (sustainable energy) niche development carried by local networks of actors, can be strategically managed and translated into the socio-technical regime is particularly compelling and offers a fertile ground for innovation in our spatial planning approaches. This corresponds quite well with the grounding perspective of this paper, which is determined rather by our niche-based approach and its emphasis on a local-regional interface - a smart city scale. In other words, the local level resembles the micro level of niches, which are

${ }^{5}$ Niches can be considered as relatively protected "experimental settings" or "incubators" for innovation. Niches can be part of the higher scale level (regime), located on the periphery or outside of the existing regime/system (Kemp et al. 1998; Rotmans et al. 2001b).

${ }^{6}$ At the regime level, patterns of institutions, culture, practices, lifestyles, artefacts, rules and norms are aligned in a coherent and self-reinforcing way in order to perform economic and social activities (Berkhout et al. 2004). Regime refers to the dominant culture, structure and practice embodied by physical and immaterial infrastructures patterns of institutions (D. Loorbach 2007b).

${ }^{7}$ Socio-technical landscape consists of autonomous trends, paradigms and slow changes (e.g. geo-political dynamics, macroeconomic trends etc.) that are beyond the direct influence of a regime or niches. Therefore, landscape typically develops autonomously and changes more slowly, however, influences the dynamics at the lower levels (regime and niches) (D. Loorbach 2007b). An example of a destabilizing element in the landscape is climate change.

8 We can follow the trend of moving from centralized energy systems towards alternative practices involving non-conventional electricity generation systems characteristic for decentralized energy generation and supply, which are becoming increasingly attractive options (Ackermann et al. 2001; Alanne \& Saari 2006; Loorbach et al. 2008; Wolsink 2014). 
situated at the base of a multi-level system, beneath incumbent socio-technical regimes and overarching landscapes (Smith 2007, p.1). In this regard, the recognition of energy as a spatially determined socio-technical system is crucial. Embracing the niche-based perspective also implies to adopt a contextual, place-based approach in spatial planning. This concerns particularly our desire to support mapping and building of indigenous innovation capabilities (through learning, networking etc.) in the field of sustainable energy and to facilitate their spatial and socio-economic integration through the means of spatial planning. Indeed, this work embraces the idea that "sustainable energy niches" become much more meaningful:

- when niches and other energy initiatives make use of their unique contexts and its special qualities (territorial capital) ${ }^{9}$,

- when they promote integration and sustainability - they should be based on an understanding of local needs, conditions, dynamics and potentials, and that includes local residents and stakeholders in a collaborative planning process,

- when they activate area-based linkages from which both niches as well as the physical and socio-economic landscape in which they are located can benefit (synergy effects) - processes of co-evolution can be stimulated.

- when they have the ambition of system innovations at higher levels.

From this perspective, niches develop largely within the constraints endowed by existing regional assets, however, their broader integration into the territory can eventually press for "reforms" at the regime level. In this regard, "better" spatial and socio-economic integration of niches might also create a window of opportunity for optimisation of renewable energy value chain and second-order learning between niches, empowering them (niche networking) and, in some cases, allows for their upscaling and diffusion. In addition to the focus of TM to attend to the dynamic patterns at and between different scale levels of socio-technical system, the proposition to entail the dimension of space in relation to the area-specific conditions of niches is in place. Given the contexts sketched above, the area-based framing of niches allows to make use of local or regional assets and advantages and hence also recognizes the multi-functional potential of sustainable energy developments (e.g. in relation to regional development). In other words, the ability of niches to "valorise" their unique context can be understood as one of the main drivers of their innovative capacity. The latter is a key point in relation to spatial planning, since the area-based understanding of niches reveals the potential to frame and conceptualize both innovation and integration of the energy initiatives in a more integrative manner - as spatially and socio-economically embedded phenomena. On this basis, the core assumption here is that if such "sustainable energy" niches were constructed appropriately, they would also have manifold synergy-effects towards sustainable spatial development.

\footnotetext{
9 Territorial capital can be described as the system of territorial assets of economic, cultural, social and environmental nature that ensures the development potential of places. (Camagni 2008).
} 


\section{Conclusions}

Bluntly stated, the challenge for further research should are the niches, their development and co-evolution with their local and supra-local context in order to explore how to utilize bottom-up renewable energy developments in a more strategic way and optimize their value chain. Although the niche-based approach is built upon the TM framework, it differs in its focus since the TM deals with the management of the whole system. However, such bottom-up approach is limited in terms of its bias towards niche-driven processes, meaning that niche pressures on the regime are overly articulated. Therefore, attention needs to be paid also to ongoing processes at the regime and landscape level and subsequent reflection upon their spatial implications is essential. Indeed, niches can be incorporated into the existing regime and thus support it or even improve it. Moreover, we should not be blind about the possibility of innovations coming directly from the incumbent regime or other regimes. The role of both bottom-up and top-down influences that shape the regime needs to be incorporated in our considerations. However, all societal actors exert influence on transitions but no single actor is not capable to control the pace and direction of transitions entirely (Rotmans et al. 2001b). Therefore, a balance between top-down and bottom-up elements within our approach to TM will be needed ${ }^{10}$. We also need to take into account that TM is a multifaceted research topic which has still a long way to go. The TM as both theoretical framework and exercise is still in its "beginnings" in the sense that there are still many opportunities to interrogate, revise and validate its perspectives before it can be considered part of main-stream science and applied with greater confidence (Genus \& Coles 2008; Geels 2011). Nevertheless, relatively robust foundations of the TM have been already laid and even empirically applied to several ongoing projects in different contexts. On this basis, a strong conclusion arises from the literature: the empirically driven methodology and integrative framework of TM can inform and assist a broad range of scientific disciplines as well as can be promoted as a potential guidance for planners and policy makers to learn and understand how they can contribute to the transition towards sustainable energy systems and hence towards smart city initiatives in a more structured, coordinated and sophisticated way at multiple levels.

\section{Acknowledgement}

This contribution is the result of the project implementation: SPECTRA+ No. 26240120002 "Centre of Excellence for the Development of Settlement Infrastructure of Knowledge Economy" supported by the Research \& Development Operational Programme funded by the ERDF.

\footnotetext{
${ }^{10}$ However, some authors also point out the shortcomings of the TM in relation to its empirical applications. Concerns associated with the inconsistency and lack of empirical evidence for the validation of TM framework as well as difficulties linked to the delineation, operationalization and specification of respective conceptual levels of niches, regimes and landscapes in practical applications have been discussed elsewhere (Berkhout et al. 2004; Genus \& Coles 2008).
} 


\section{References}

Ackermann, T., Andersson, G. \& Söder, L., 2001. Distributed generation: a definition1. Electric Power Systems Research, 57(3), pp.195-204. Available at: http://www.sciencedirect.com/science/article/pii/S0378779601001018.

Alanne, K. \& Saari, A., 2006. Distributed energy generation and sustainable development. Renewable and Sustainable Energy Reviews, 10(6), pp.539-558.

Araújo, K., 2014. The emerging field of energy transitions: Progress, challenges, and opportunities. Energy Research \& Social Science, 1, pp.112-121. Available at: http://www.sciencedirect.com/science/article/pii/S2214629614000164.

Bagliani, M., Dansero, E. \& Puttilli, M., 2010. Territory and energy sustainability: the challenge of renewable energy sources. Journal of Environmental Planning and Management, 53(4), pp.457-472. Available at: http://www.tandfonline.com/doi/abs/10.1080/09640561003694336 [Accessed December $15,2014]$.

Basta, C., van der Knaap, W. \& Carsjens, G.J., 2012. Planning Sustainable Energy Landscapes: From Collaborative Approaches to Individuals' Active Planning. Sustainable Energy Landscapes: Designing, Planning, and Development, 7, p.187.

Berkhout, F., Smith, A. \& Stirling, A., 2004. Socio-technological regimes and transition contexts. System innovation and the transition to sustainability: theory, evidence and policy. Edward Elgar, Cheltenham, 44(106), pp.48-75.

Bridge, G. et al., 2013. Geographies of energy transition: Space, place and the low-carbon economy. Energy Policy, 53, pp.331-340. Available at: http://dx.doi.org/10.1016/j.enpol.2012.10.066.

Camagni, R., 2008. Regional competitiveness: towards a concept of territorial capital. In Modelling regional scenarios for the enlarged Europe. Springer, pp. 33-47.

Commission, C.F. the \& the Commission, C.F., 2010. Europe 2020: a strategy for smart, sustainable and inclusive growth. Brussels: European Commission. Available at: $\mathrm{http} / / /$ scholar.google.com/scholar?hl=en\&btnG=Search\&q=intitle:A+strategy+for+smart ,+sustainable+and+inclusive+growth $\# 0$.

Davoudi, S., 2009. Framing the Role of Spatial Planning in Climate Change. Electronic Working Paper, 43(43), pp.1-44.

Davoudi, S., Crawford, J. \& Mehmood, A., 2009. Climate change and spatial planning responses. Planning for climate change: strategies for mitigation and adaptation for spatial planners, pp.1-18.

European Commission, 1999. European spatial development perspective, Available at: $\mathrm{http} / /$ ec.europa.eu/regional_policy/sources/docoffic/official/reports/pdf/sum_en.pdf.

European Innovation Partnership, 2013. European Innovation Partnership on Smart Cities and Communities Strategic Implementation Plan. European Innovation Partnership on Smart Cities 2013, (Strategic Implementation Plan).

Geels, F.W., 2011. The multi-level perspective on sustainability transitions: Responses to seven criticisms. Environmental innovation and societal transitions, 1(1), pp.24-40.

Geels, F.W. \& Schot, J., 2007. Typology of sociotechnical transition pathways. Research policy, 36(3), pp.399-417.

Genus, A. \& Coles, A.-M., 2008. Rethinking the multi-level perspective of technological transitions. Research policy, 37(9), pp.1436-1445.

Giordano, V. et al., 2011. Smart Grid projects in Europe: lessons learned and current developments,

IPCC, 2014. Climate Change 2014 Synthesis Report Summary Chapter for Policymakers. Ipcc, p.31.

Jiusto, S., 2009. Energy Transformations and Geographic Research. In A Companion to Environmental Geography. Wiley-Blackwell, pp. 533-551. Available at: 
http://dx.doi.org/10.1002/9781444305722.ch31.

Kemp, R. \& Loorbach, D., 2006. 5. Transition management: a reflexive governance approach. Reflexive Governance for Sustainable Development, Cheltenham, UK and Northampton, MA, USA: Edward Elgar, pp.103-130.

Kempener, R., Komor, P. \& Hoke, A., 2013. Smart Grids and Renewables - A Guide for Effective Deployment. International Renewable Energy Agency, (November), p.47.

Loorbach, D. a, 2010. Transition Management for Sustainable Development: A Prescriptive, Complexity-Based Governance Framework. Governance, 23(1), pp.161-183. Available at: http://doi.wiley.com/10.1111/j.1468-0491.2009.01471.x.

Loorbach, D., Van Der Brugge, R. \& Taanman, M., 2008. Governance in the energy transition: Practice of transition management in the Netherlands. International Journal of Environmental Technology and Management, 9(2-3), pp.294-315.

Nations, U., 2008. SPATIAL PLANNING - Key Instrument for Development and Effective Governance with Special Reference to Countries in Transition. , pp.1-56. Available at: http://www.unece.org/fileadmin/DAM/hlm/documents/Publications/spatial_planning.e.p df.

Pasqualetti, M.J., 2011. Social barriers to renewable energy landscapes*. Geographical Review, 101(2), pp.201-223.

De Roo, Hillier, J. \& Van Wezemael, J., 2012. Complexity and spatial planning: introducing systems, assemblages and simulations. Complexity and Spatial Planning: Systems, Assemblages and Simulations, pp.1-32.

Rotmans, J., Kemp, R. \& Van Asselt, M., 2001a. More evolution than revolution: transition management in public policy. Foresight, 3(1), pp.15-31.

Rotmans, J., Kemp, R. \& Van Asselt, M., 2001b. More evolution than revolution: transition management in public policy. foresight, 3(1), pp.15-31.

Rotmans, J. \& Loorbach, D., 2008. Transition management: reflexive governance of societal complexity through searching, learning and experimenting, Edward Elgar: Cheltenham, UK.

Smith, A., 2007. Translating sustainabilities between green niches and socio-technical regimes. Technology Analysis \& Strategic Management, 19(4), pp.427-450.

Smith, A., Voß, J.-P. \& Grin, J., 2010. Innovation studies and sustainability transitions: The allure of the multi-level perspective and its challenges. Research policy, 39(4), pp.435448.

Solomon, B.D., Pasqualetti, M.J. \& Luchsinger, D.A., 2003. Energy geography. Geography in America at the dawn of the 21st century. Oxford, pp.302-313.

Stremke, S. \& van den Dobbelsteen, A., 2012. Sustainable energy landscapes: designing, planning, and development, CRC Press.

Wolsink, M., 2014. Distributed generation of sustainable energy as a common pool resource: social acceptance in rural setting of smart (micro-) grid configurations.

Wolsink, M., 2013. Distributed generation of sustainable energy as a common pool resource: social acceptance in rural settings of smart (micro-) grid configurations. 